\title{
FRICTION, WEAR AND CORROSION: LEARNING FROM NATURE
}

\author{
V. STOILOV \& D. O. NORTHWOOD \\ Department of Mechanical, Automotive and Materials Engineering, University of Windsor, Canada.
}

\begin{abstract}
Friction, wear and corrosion play a central role in diverse systems and phenomena that at first sight may seem unrelated. On closer scrutiny, however, bio-system phenomena such as the lotus leaf effect (hydrophobicity) and surface passivation are found to display common features that are shared by many tribological processes in technological (manufacturing and automotive) and geological (drilling and mining) applications. Through the process of natural selection, nature has produced surface textures and water-based lubricant systems that far outclass the best oil-based lubricants of most man-made devices. To emulate these systems is one of today's great challenges.

Keywords: Corrosion resistance, hydrophobicity, surface patterning, ultralow friction.

\section{INTRODUCTION}

Many terrestrial plants and animals are water-repellent due to hydrophobic surface components with microscopic roughness. These surfaces provide a very effective anti-adhesive property against particulate contamination. This self-cleaning mechanism, called the 'Lotus-Effect', is an important function of many micro-structured biological surfaces [1]. It is now recognized that the fascinating fluid behaviors observed for the lotus plant, like the rolling and bouncing of liquid droplets and selfcleaning of particle contaminants, arise from a combination of the low interfacial energy and the rough surface topography of waxy deposits covering their leaves [2].
\end{abstract}

\section{NATURE'S SOLUTIONS TO CONTROL FRICTION, WEAR AND CORROSION}

\subsection{Hydrophobic surfaces}

As shown in Fig. 1, the surface of the lotus leaf is covered with micro-protrusions, which are clothed in nano-protrusions. The nano-protrusions are composed of epicuticular wax crystalloids that are hydrophobic [3].
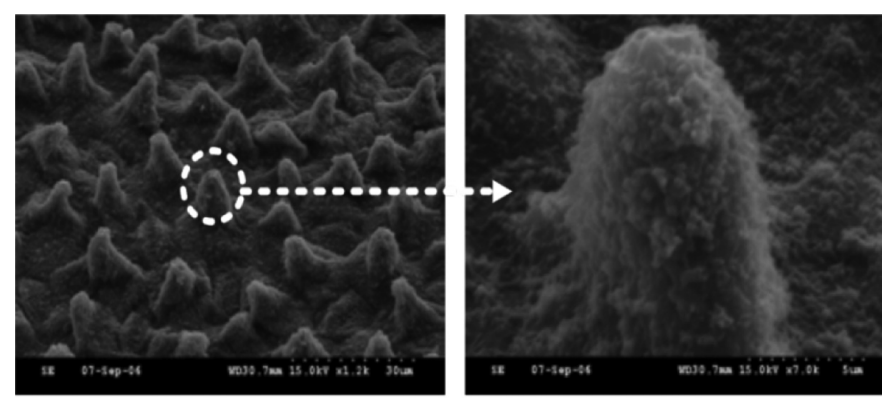

Figure1: SEM images of the surface of a lotus leaf [3]. 
The unusual wetting characteristics of superhydrophobic surfaces are governed by both their surface chemical composition and surface geometric microstructure [4,5]; their wettability can be decreased by creating a local geometry with a large geometric area relative to the projected area [4]. The origin of the self-cleaning property of lotus leaves has been revealed to be a cooperative effect of micro- and nano-scale structures on their surfaces [5]. Cheng and his colleagues [6] emphasized the importance of the lotus leaf's nanoscale hair-like structure on its self-cleaning ability. Barthlott et al. assumed that 'Lotus effect' can be transferred to artificial surfaces (e.g. cars, facades, foils) and hence find an immense number of technical applications [1]. As the surface free energy of a solid surface decreases, hydrophobicity increases. Wetting properties are defined by the magnitude of contact angle. If the contact angle is lower than $90^{\circ}$, a material is hydrophilic (wettable), otherwise it is hydrophobic (non or low wettable). The contact angle is not only material property dependent, but it changes with the surrounding conditions, time and it also depends on the history of wetting [7].

The apparent contact angle $(\theta)$ between a rough surface and a liquid droplet can be determined using

$$
\cos (\theta)=r \cos \left(\theta_{T}\right)
$$

where $r$ is the roughness ratio (the actual surface area divided by the apparent surface area) and $\theta_{T}$ is the thermodynamic contact angle defined by

$$
\cos \left(\theta_{T}\right)=\frac{\gamma_{\mathrm{sv}}-\gamma_{\mathrm{sl}}}{\gamma_{\mathrm{lv}}}
$$

where $\gamma_{\mathrm{sv}}$ is the solid-vapor surface energy, $\gamma_{\mathrm{sl}}$ is the solid-liquid interfacial energy and $\gamma_{\mathrm{lv}}$ is the liquid-vapor surface energy (see Fig. 2). As roughness increases, air can be locally trapped underneath the liquid, resulting in the formation of a composite surface with a large contact angle, a phenomenon that is described using the following theoretical equation:

$$
\cos (\theta)=f_{s} \cos \left(\theta_{T}\right)-f_{\text {air }},
$$

where $f_{\mathrm{S}}$ is the fractional contact area between the liquid and the solid surface, and $f_{\text {air }}$ is the fractional contact area between the liquid and the air underneath the droplet [8].

The successful advancement of micro-electro-mechanical systems (MEMS and NEMS [3,8]) with miniature moving parts, including micromotors, gears and transmissions, mechanical discriminators and optical microswitches, relies on the development of new wear resistant materials and surfaces with high hydrophobicity (water repellency) and low adhesion and friction. Other possible applications for durable water repellent surfaces range from micro-fluidic devices to bipolar plates

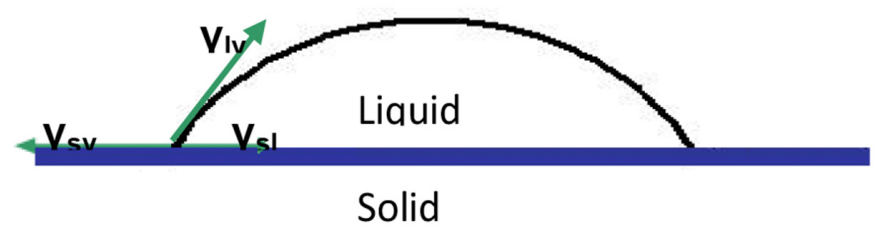

Figure 2: The schematic illustration of surface tensions on a solid surface in contact with a water droplet, where $\gamma_{\mathrm{sv}}$ is the solid-vapor surface energy, $\gamma_{\mathrm{sl}}$ is the solid-liquid interfacial energy and $\gamma_{\mathrm{lv}}$ is the liquid-vapor surface energy. 
in proton exchange membrane (PEM) fuel cells $[8,9]$. This effect inspired the application of a similar mechanism in the solid-water-corrosive systems, namely the prevention of corrosion by repelling the corrosive electrolyte from a surface, making them easily roll off by an external force.

\subsubsection{Hydrophobicity of metals}

Superhydrophobic surfaces are of great importance for many industrial applications and may present a solution to the long-standing problems of environmental contamination and corrosion of metals [10]. The prospect of producing surfaces that repel water suggests huge opportunities in the area of corrosion inhibition for metal components, chemical and biological agent protection for clothing, antifouling for marine vehicles, among many other applications [6].

Generally, metals are hydrophilic or wettable. To create a hydrophobic surface on a metal, there are some methods such as creation of surface patterns, using some chemicals and a combination of surface patterns and chemicals. The two main methods of applying sufficient pattern are etching a metal substrate and electroless deposition of a metal coating onto the substrate. Other methods that produce metal-based superhydrophobic surfaces include sulfur treatment, either with sulfur gas or direct mixing of a thiol solution with a metal $(\mathrm{Cd}$ or $\mathrm{Zn})$ salt solution. Electrochemical methods to provide roughness have also been reported for indium tin oxide (ITO)-coated glass and doped silicon. A range of techniques including anodization has been successfully applied to Al. Between presented reports, there are two different approaches to lowering the surface energy of the roughened surfaces: the use of fluorinated silanes or of fluorinated thiols. For metals, the range of compounds that could be used to lower the surface energy is much larger since there are numerous functional groups that bind to metals. Despite the diversity of approaches, which have been applied to creating superhydrophobic metal surfaces, there are just two factors, which dominate the ultimate performance. One is the roughness or texture of the surface, and the second is the nature of the surface modifying layer [6]. Generally, metal oxides are more hydrophobic than the metal, so the wettability may become lower with increase in the amount of metal oxide $[11,12]$. The weaker wetting properties of aluminum, brass and stainless steel can be explained by the process of passivation, e.g. covering with the thin layer of oxide. The zinc, aluminum or chromium oxides are hydrophobic. The passivation of copper is not such a rapid process as it is in the case of aluminum, zinc or chromium, so the surface of copper plate remains hydrophilic [7].

$\mathrm{Wu}$ and his collaborators fabricated stainless steel-based superhydrophobic surfaces by microstructuring using a femtosecond laser and the method of silanization [13]. They proved that these micro- and submicron double-scale structure surfaces yield apparent contact angles higher than those on single-scale structure surfaces, and the maximum value was $166.3^{\circ}$. Also, their results confirm that the fraction of air between the water droplet and the double-scale surface was large, which is the important reason of superhydrophobicity of the surface (Fig. 3). This provides a simple and easily controlled method for fabricating stainless steel-based superhydrophobic surfaces [13].

Nickel-based alloys have been studied and used as biomaterials due to their corrosion resistance and excellent metallurgical compatibility with alloying elements. Shi et al. have studied the tribochemical behavior of nickel sheets on exposure to a mixture of biomaterial solutions (cell culture media). The nickel sheets used in this research were designed as filters (see Fig. 4). They found that the droplet contact angle of the Ni sample is $128.3^{\circ}$, which shows the dry nickel samples are hydrophobic [16].

Hexagonal-shaped nickel micromeshes (see Fig. 5) were designed and fabricated and their water-repellent and water-proofing abilities were investigated by Lee and his collaborators [3]. 
(a)

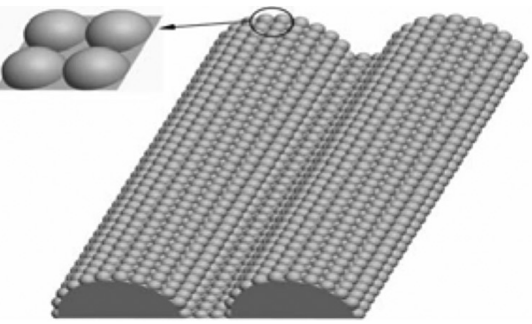

(b)

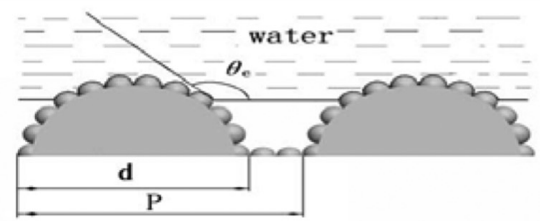

Figure 3: Schematic illustration of laser-induced periodic surface structure (LIPSS) covered with nanoparticles formed at fluences $0.08 \mathrm{~J} / \mathrm{cm}^{2}$. (a) The alternating array of LIPSS and nanoparticles. (b) The local interaction of the sort water interface with the top of LIPSS [13].
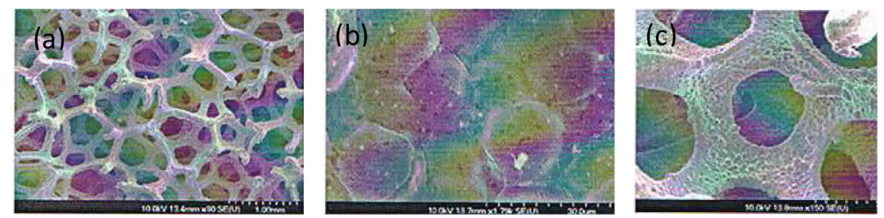

Figure 4: Surface and microstructure of Ni; SEM images: (a) overview, (b) pentagon and (c) pore [16].

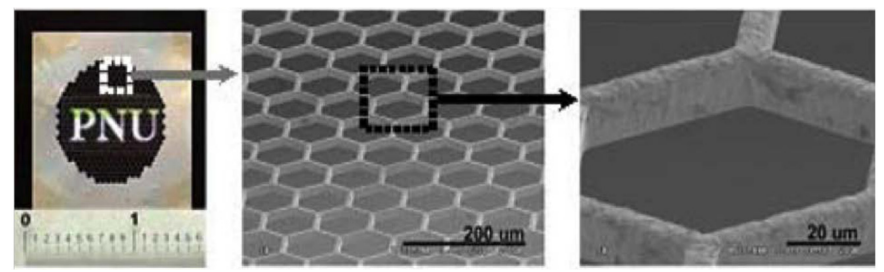

Figure 5: Fabricated nickel micromesh sheet: (left) photograph of a nickel micromesh prototype unit, (middle and right) magnified SEM images of the micromesh [3].

By the effect of the micromesh and PPFC coating, the contact angles were increased sharply from $63^{\circ}$ of the non-coated flat nickel to $140^{\circ}$ of the PPFC-coated micromesh to modify the surface from hydrophilic to superhydrophobic. The hole size and increasing the lattice width of the micromesh are necessary to improve the water-proofing ability. The proposed nickel micromesh sheets can be applied to many application areas that require water-blocking yet must allow passage of gases or sound waves. Examples include water-proof phones/speakers and water-proof gas detectors [3].

Lotus leaf surface-textured nanocrystalline (NC) Ni films, developed by replicating the original biotexture, modified using a selective electrodeposition and a PFPE solution treatment were fabricated by Shafiei and Alpas (see Fig. 6). The superhydrophobicity of the NC Ni films is attributed to successful development of a multi-level surface roughness (where a nanoscale surface texture was superimposed on a microscale structure of protuberances) with a low surface energy [8]. 


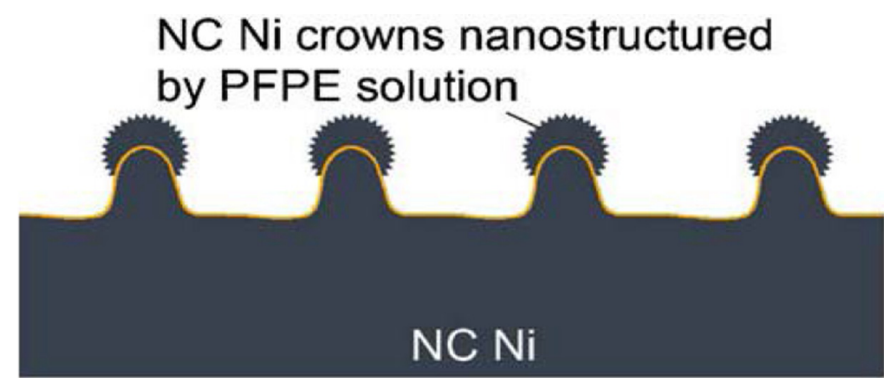

Figure 6: A PFPE solution treatment of the surface created a nanotextured layer on the "Ni crowns" [8].

\section{LESSONS LEARNED FROM NATURE}

\subsection{Corrosion control through surface patterning and roughness}

The challenge in forming heterogeneous interfaces from hydrophilic materials lies in designing surface topographies that will lead to stable air/vapor entrapment. There are two main parameters that are important for creating heterogeneous solid-liquid interfaces. One is the topology of the surface and the other is the nature of the surface layer. The mechanism of roughness-induced heterogeneous wetting is complicated and involves effects over various length scales. The composite interface is fragile, since transition to a homogeneous interface is irreversible, and therefore the stability of a composite interface is crucial and should be addressed for the successful development of corrosion-resistant surfaces.

A patterned surface with composite heterogeneous solid-liquid-air interface is notable as it has the features of liquid repellency and low surface energy. The effect of surface patterning on the corrosion behavior of a metal (nickel) was investigated [17-19]. The idea originates in the 'lotus leaf' effect and it is based on the fact that hydrophobic (low or non-wettable) surfaces can decrease the contact area between a corrosive solution and a surface. Special surface patterns with similar to the lotus leaf micro-texture were created on pure nickel sheets (Fig. 7). The corrosion behavior of those surfaces was investigated [14-19] by using a dynamic polarization method in $\mathrm{H}_{2} \mathrm{SO}_{4}$ solution. All patterned samples showed vastly superior corrosion resistance compared with a reference smooth polished sample [17]. A further investigation (EDS and SEM) in the nature of the formed electrolyte-metallic interface on the patterned samples showed the formation of heterogeneous wetting, characteristic for hydrophobic surfaces [17].

To clearly underline the hydrophobic nature of the patterned surfaces, their corrosion resistance was compared with similar size unidirectional random roughness on nickel surfaces [18]. A smoother surface in the case of the unidirectional rough surfaces will result in lower corrosion rate (Fig. 8). The introduction of the unidirectional random roughness effectively increases the wettability of the surface, which in turn increases the area of the contact between the electrolyte and the metal surface, and leads to increase in the corrosion rate. In contrast, the patterned surfaces have the highest roughness (Fig. 8) but showed the smallest corrosion rate compared with the unidirectional random roughness samples. This result is consistently repeated in the roughness measurements, EIS analysis, potentiodynamic polarization tests and SEM images [18]. 

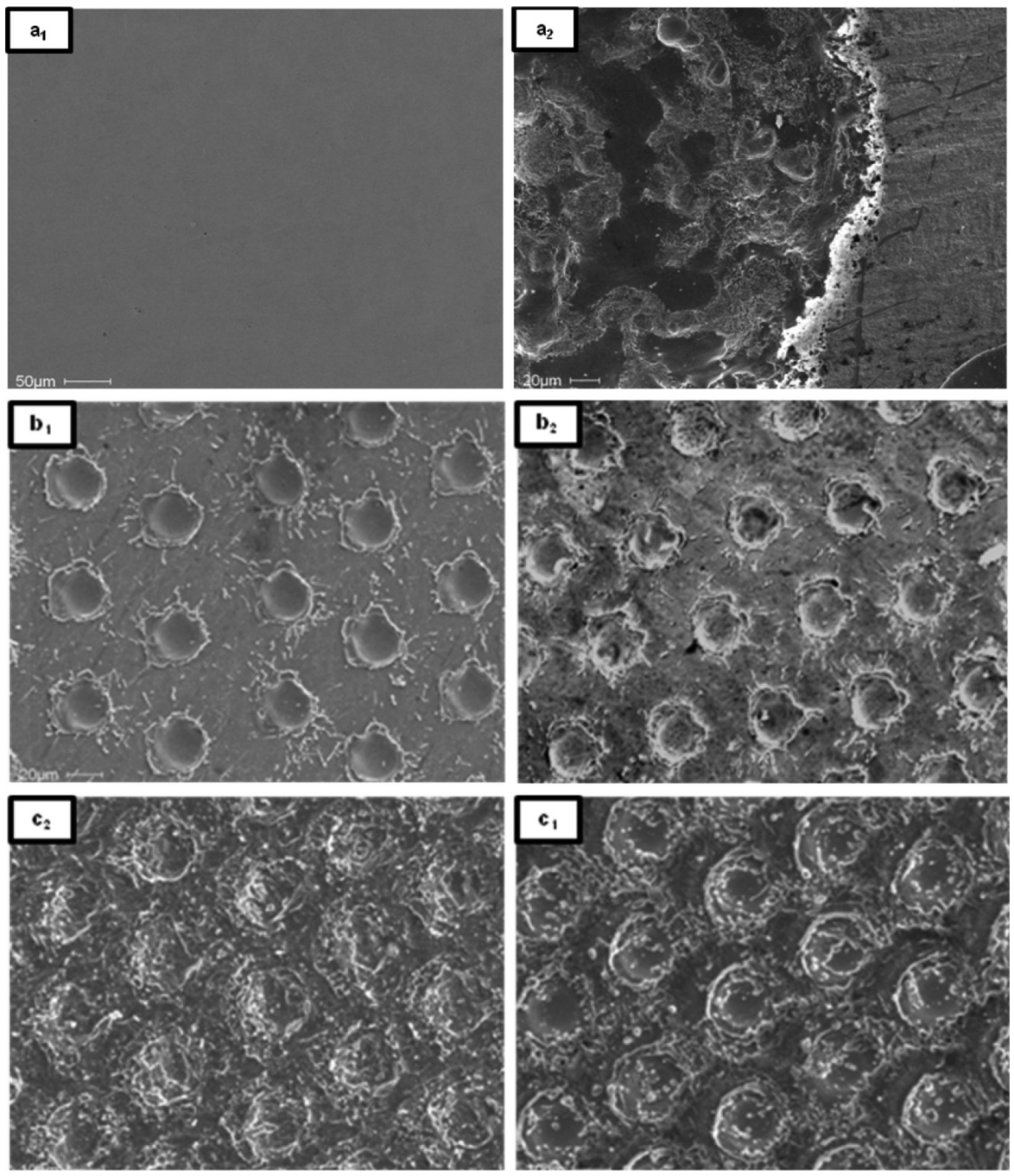

Figure 7: SEM of the nickel sheets before and after the corrosion test. $\left(a_{1}\right)$ Reference sample before corrosion test. $\left(\mathrm{a}_{2}\right)$ Reference sample after corrosion test. $\left(\mathrm{b}_{1}\right)$ Sample D30L60 after corrosion test. $\left(b_{2}\right)$ Sample D30L60 after corrosion test. $\left(c_{1}\right)$ Sample D20L30 before corrosion test. $\left(\mathrm{c}_{2}\right)$ Sample D20L30 after corrosion test [18].

\subsection{Hydrophobicity in tribology}

In addition to improving corrosion resistance, the surface texturing has gained momentum as a way to control the friction, which is involved in various applications and systems, such as components of internal combustion engines, dies and punches of the metal-forming processes and MEMS.

Under dry friction, the area of contact is the crucial parameter that controls both the mechanical and adhesive components of the friction force. This why, mimicking the 'lotus leaf' surface texture to engineer the real area of contact is a promising approach to control friction. Analytically, it has 


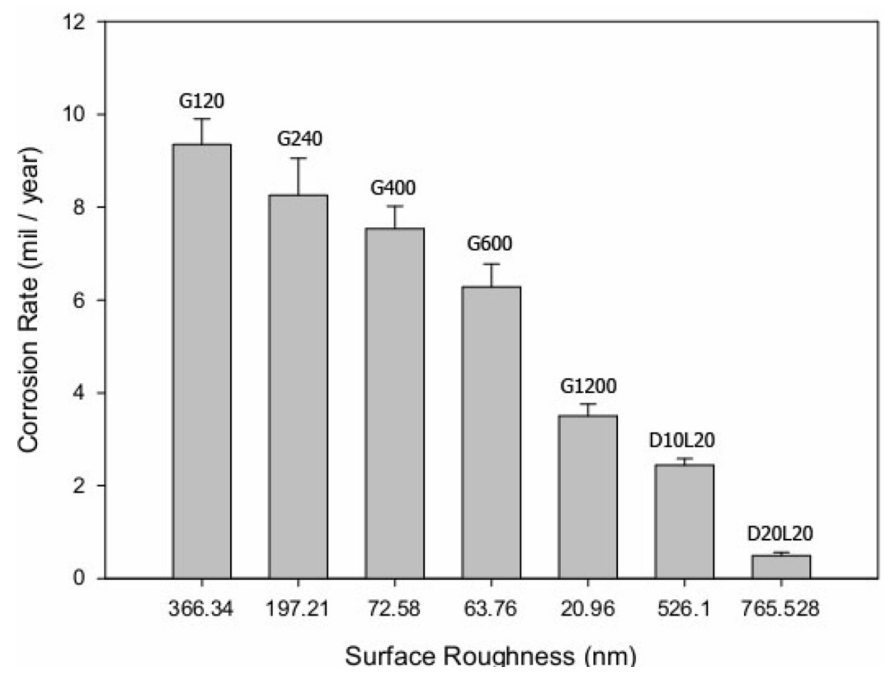

Figure 8: Corrosion rate values for different surface roughnesses.

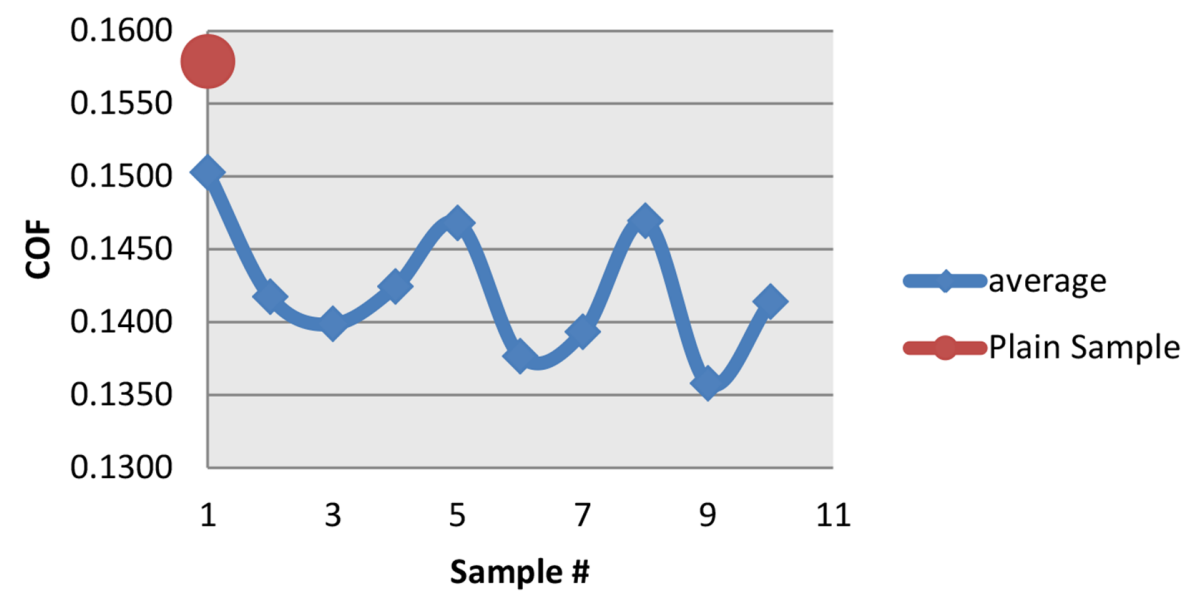

Figure 9: Friction coefficient of the reference sample and all patterned samples (error of 2.5\%).

been proven that friction can be minimized at specific real contact area [20,21]. In two- and threedimensional finite element models, normal and sliding contact between a rigid indenter and elastic-plastic surfaces, which are textured by circular and hexagonal dimples of different sizes and densities, are simulated and analyzed [21]. Circular craters of different sizes and densities are fabricated using laser ablation on hardened tool steel samples, whereas the hexagonal dimples are fabricated using photo-lithography. The dimples are arranged in adjacent equilateral triangles layout. Coefficients of friction were measured using a scratch tester under dry sliding conditions and constant load.

Figure 9 displays the average coefficient of friction of each sample that has different dimple sizes (D) and different spacings between the dimples (L). The plain sample has the highest COF, 
Table 1: Coefficient of friction and its relative reduction in plain and patterned surfaces.

\begin{tabular}{lccccccccccc}
\hline $\begin{array}{l}\text { Sample } \\
\#\end{array}$ & & 1 & 2 & 3 & 4 & 5 & 6 & 7 & 8 & 9 & 10 \\
\hline Average & 0.158 & 0.150 & 0.1417 & 0.140 & 0.142 & 0.147 & 0.138 & 0.139 & 0.147 & 0.136 & 0.141 \\
Std dev. & 0.027 & 0.026 & 0.023 & 0.024 & 0.024 & 0.026 & 0.023 & 0.024 & 0.024 & 0.022 & 0.026 \\
Red \% & 0.000 & 4.815 & 10.231 & 11.411 & 9.784 & 7.018 & 12.809 & 11.763 & 6.930 & $\mathbf{1 3 . 9 9 6}$ & 10.432 \\
\hline
\end{tabular}

surpassing the COF of any patterned samples. The maximum friction reduction achieved with the selected patterning is $\sim 14 \%$ (Table 1 ). This clearly shows that under dry sliding contact condition, the selected textures reduce friction. This is mainly due to the reduction of the contact area, which in turn leads to reduction in the adhesion component of the friction force. Also, the surface dimples created due to patterning entrap any wear debris, and therefore reduce the number of the asperities that interlock with the counter surface. The overall result is less plowing force and less plastic deformation.

In addition, adhesion forces, measured using an atomic force microscope (AFM), showed exponential decrease with the increase in the spatial texture density. The dimensionless spatial texture density $(D / L)$ defined as the ratio of the size of the crater $(D)$ to the distance between the centers of two consecutive craters $(L)$, was identified to be the most significant texturing parameter. A minimum coefficient of friction exits at values of spatial texture densities that lie between 0.25 and 0.5 .

\section{CONCLUDING REMARKS}

The unique functionality of the surface patterning, inspired by the 'lotus leaf' effect, has been described in an attempt to draw out some of the potential for biomimetic-based design in corrosion protection and tribology. The paper discusses the potential strategies to mimic the functionality of the 'lotus leaf' and identifies fruitful research areas to achieve the potential gains from a biomimetic approach in tribology and corrosion protection.

\section{REFERENCES}

[1] Barthlott, W. \& Neinhuis, C., Purity of the sacred lotus, or escape from contamination in biological surfaces. Planta, 202, pp. 1-8, 1997. doi: http://dx.doi.org/10.1007/s004250050096

[2] Barkhudarov, P.M., Shah, P.B., Watkins, E.B., Doshi, D.A. Brinker, C.J. \& Majewski, J., Corrosion inhibition using superhydrophobic films. Corrosion Science, 50, pp. 897-902, 2008. doi: http://dx.doi.org/10.1016/j.corsci.2007.10.005

[3] Lee, S.M., Oh, D.J., Jung, I.D., Bae, K.M., Jung, P.G., Chung, K.H., Cho, S.J. \& Ko, J.S., Fabrication of nickel micromesh sheets and evaluation of their water-repellent and waterproof abilities. International Journal of Precision Engineering and Manufacturing, 10(3), pp. 161166, 2009. doi: http://dx.doi.org/10.1007/s12541-009-0062-8

[4] Han, J.T., Jang, Y., Lee, D.Y., Park, J.H., Song, S.H., Banb, D.Y. \& Cho, K., Fabrication of a bionic superhydrophobic metal surface by sulfur-induced morphological development. Journal of Materials Chemistry, 15, pp. 3089-3092, 2005. doi: http://dx.doi.org/10.1039/b504850h

[5] Jin, M., Feng, X., Xi, J., Zhai, J., Cho, K., Feng, L. \& Jiang, L., Super-hydrophobic PDMS surface with ultra-low adhesive force. Macromolecular Rapid Communications, 26, pp. 18051809, 2005. doi: http://dx.doi.org/10.1002/marc.200500458 
[6] Gajewski, A., Contact angle and sessile drop diameter hysteresis on metal surfaces. International Journal of Heat and Mass Transfer, 51, pp. 4628-4636, 2008. doi: http://dx.doi. org/10.1016/j.ijheatmasstransfer.2008.01.027

[7] Liu, T., Lau, K.T., Chen, S., Cheng, S. \& Yin, Y., Super-hydrophobic surfaces improve corrosion resistance of $\mathrm{Fe}_{3} \mathrm{Al}$-type intermetallic in seawater. Advanced Materials Research, 47-50, pp. 173-176, 2008. doi: http://dx.doi.org/10.4028/www.scientific.net/amr.47-50.173

[8] Shafiei, M. \& Alpas, A.T., Nanocrystalline nickel films with lotus leaf texture for superhydrophobic and low friction surfaces. Applied Surface Science, 256, pp. 710-719, 2009. doi: http:// dx.doi.org/10.1016/j.apsusc.2009.08.047

[9] Lee, S.J., Huang, C.H., Lai, J.J. \& Chen, Y.P., Corrosion-resistant component for PEM fuel cells. Journal of Power Sources, 131, pp. 162-168, 2004. doi: http://dx.doi.org/10.1016/j. jpowsour.2004.01.008

[10] Liu, T., Yin, Y. \& Dong, L., New application of the underwater super-hydrophobic surface in the corrosion protection. Advanced Materials Research, 79-82, pp. 1115-1118, 2009. doi: http:// dx.doi.org/10.4028/www.scientific.net/amr.79-82.1115

[11] Wang, R. \& Kido, M., Corrosion behavior of pure iron beneath a micro-droplet of sulfuric acid solution investigated by atomic force microscopy. Scripta Materialia, 55, pp. 633-636, 2006. doi: http://dx.doi.org/10.1016/j.scriptamat.2006.06.011

[12] Zhu, L. \& Jin, Y., A novel method to fabricate water-soluble hydrophobic agent and superhydrophobic film on pretreated metals. Applied Surface Science, 253, pp. 3432-3439, 2007. doi: http://dx.doi.org/10.1016/j.apsusc.2006.07.050

[13] Wu, B., Zhou, M., Li, J., Ye, L., Li, G. \& Cai, L., Superhydrophobic surfaces fabricated by microstructuring of stainless steel using a femtosecond laser. Applied Surface Science, 256, pp. 61-66, 2009. doi: http://dx.doi.org/10.1016/j.apsusc.2009.07.061

[14] Larmour, I.A., Saunders, G.C. \& Bell, S.E.J., Assessment of roughness and chemical modification in determining the hydrophobic properties of metals. New Journal of Chemistry, 32, pp. 1215-1220, 2008. doi: http://dx.doi.org/10.1039/b718006c

[15] Kurtz, O., Barthelmes, J., Broc, F.L., Bilkay, T., Danker, M. \& Ruther, R., Gold post-dip to improve corrosion-resistance properties. Metal Finishing, 1, pp. 24-30, 2009. doi: http://dx.doi. org/10.1016/s0026-0576(09)00028-2

[16] Shi, B., Kuhn, T.B., Liang, H. \& Duffy, L.K., Tribochemical performance of cell-treated nickel matrix. American Journal of Biotechnology and Biochemistry, 3, pp. 141-144, 2007. doi: http://dx.doi.org/10.3844/ajbbsp.2007.141.144

[17] Toloei, A.S, Stoilov, V. \& Northwood, D.O., A new approach to combating corrosion of metallic materials. Applied Surface Science, 284, pp. 242-247, 2013. doi: http://dx.doi.org/10.1016/j. apsusc.2013.07.088

[18] Toloei, A.S., Stoilov, V. \& Northwood, D.O., The effect of different surface topographies on the corrosion behaviour of nickel. WIT Transactions on Engineering Science, 77, pp. 193-204, 2013. doi: http://dx.doi.org/10.2495/mc130171

[19] Bigdeli-Karimi, M., Stoilov, V. \& Northwood, D.O., Mater. Charact. V. WIT Transactions on Engineering Sciences, 72, pp. 85-93, 2011.

[20] Rashwan, O., Stoilov, V., Alpas, A. \& Guerrero, A., Effect of surface patterning on the adhesive friction. Proceedings of ASME 2012 International Mechanical Engineering Congress \& Exposition, IMECE2012-89644, 2012. doi: http://dx.doi.org/10.1115/imece2012-89644

[21] Rashwan, O. \& Stoilov, V., Numerical model of microstructure and fracture of coated aluminum alloys: a novel design approach. Materials Science Forum, 706-709, pp. 2640-2645, 2012. doi: http://dx.doi.org/10.4028/www.scientific.net/msf.706-709.2640 